\title{
A Broadly Applicable Alkyl-Alkyl Suzuki-Miyaura Cross-Coupling Reac- tion Catalyzed by an Iron-Based Complex
}

\author{
Chet C. Tyrol, Jeffery A. Byers* \\ Department of Chemistry, Merkert Chemistry Center, Boston College, Chestnut Hill, Massachusetts 02467, United States
}

\section{Supporting Information Placeholder}

\begin{abstract}
An iron-based catalyst for the Suzuki-Miyaura reaction between two $\mathrm{sp}^{3}$-hybridized substrates has been developed for a broad range of unactivated alkyl halides and alkyl boranes. Key to success was using a $C_{S}$-symmetric $\beta$ diketiminate ligand that contained a tert-butyl and trifluoromethyl functionalized backbone. The breadth of the cross-coupling reaction was demonstrated with high yields of cross-coupled products observed from reactions using primary and secondary alkyl bromides as well as primary alkyl boranes and even some secondary alkyl boranes. Complementary methylation reactions using methyl iodide or methyl-9-BBN was also possible. The facile diversification of a steroid derivative using these reactions demonstrated the synthetic utility of such complementary cross-coupling reactions.
\end{abstract}

Cross-coupling reactions have found widespread adoption among industrial medicinal and process chemists, ${ }^{1}$ and are a commonly used reaction among synthetic organic chemists in academia. ${ }^{2,3}$ Despite the proliferation of cross-coupling reactions in chemical synthesis, many of the bond formations are limited to $\mathrm{sp}^{2}-\mathrm{sp}^{2}$ and $\mathrm{sp}^{3}-\mathrm{sp}^{2}$ couplings, while $\mathrm{sp}^{3}-\mathrm{sp}^{3}$ couplings remain more elusive. ${ }^{4}$ In fact, $\mathrm{sp}^{3}-\mathrm{sp}^{3}$ examples represent only $1 \%$ of all Suzuki-Miyaura reactions reported to date. ${ }^{5}$ The challenges with $\mathrm{sp}^{3}-\mathrm{sp}^{3}$ crosscoupling stem from competitive $\beta$-hydride elimination events ${ }^{1}$ along with comparatively sluggish oxidative addition of $2^{\circ}$ and $3^{\circ}$ alkyl halide electrophiles to the palladium-based catalysts that are typically employed. ${ }^{6}$

Despite being uncommon, some palladium and nickel-based catalysts have been developed that have enabled $\mathrm{sp}^{3}-\mathrm{sp}^{3}$ Suzuki-Miyaura cross-coupling between primary alkyl boranes and primary or secondary alkyl halides. ${ }^{6-10}$ Utilizing secondary alkyl halides enabled enantioselective variants, which have been reduced to practice when suitable directing groups are installed on the electrophile.11-16 Though considerable advances have been made with nickel-based catalysts that are most commonly used for these reactions, there are many challenges that remain. Examples of alkyl-alkyl cross-coupling that involve secondary alkyl boron nucleophiles are rare; only one example has been reported. ${ }^{14}$ Additionally, limited examples exist that report cross-coupling reactions with electrophiles that contain heteroaromatic functionality, a common feature in many biologically active small molecules. ${ }^{15,17}$ Finally, methylations have not been reported.

To address these limitations, iron-based catalysts are an attractive alternative. In addition to being less toxic, ${ }^{18}$ iron-based catalysts often demonstrate fast reaction kinetics ${ }^{19}$ and rarely undergo isomerization events that sometimes complicate cross-coupling reactions catalyzed by nickel-based catalysts. ${ }^{20-22}$ Consequently, the properties of iron-based catalysts could lead to a complementary substrate scope compared to nickel-based catalysts, which we have seen with the Suzuki-Miyaura arylation of tertiary alkyl halides.5,23 Only within the last decade have iron-based crosscoupling catalysts been used for constructing $\mathrm{sp}^{3}-\mathrm{sp}^{3}$

Scheme 1: a) Previously reported iron-catalyzed $\mathrm{sp}^{3}-\mathrm{sp}^{3}$ Suzuki-Miyaura coupling. b) $\beta$-diketiminate iron-complex catalyzed Suzuki-Miyaura $\mathrm{sp}^{3}-\mathrm{sp}^{3}$ coupling reaction.

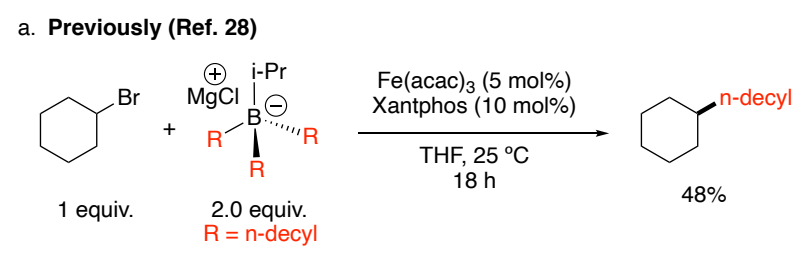

b. This work:

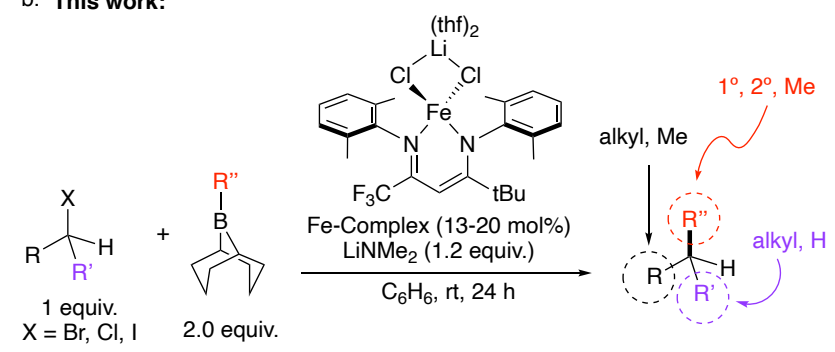


bonds, ${ }^{24-28}$ with a single report disclosing a SuzukiMiyaura reaction (Scheme 1a). ${ }^{28}$ While this example was groundbreaking because it demonstrated the applicability of iron-based catalysts for these challenging cross-coupling reactions, the reaction had a limited substrate scope. All of the reported electrophiles were alkyl bromides, and the only examples involving secondary alkyl bromides were derived from cyclic alkyl bromides containing six-membered rings. Moreover, the borate transmetalating reagents were derived from three equivalents of the nucleophilic partner, and no examples were reported for secondary alkylboranes. In this work, we demonstrate how utilizing iron complexes bearing the appropriate anionic $\beta$ diketiminate ligands lead to a broader alkyl halide and alkylborane substrate scope, including examples involving alkyl chlorides, a variety of cyclic and acyclic secondary alkyl bromides, reactions involving some secondary alkylboranes, and even methylation reactions where the methylating source is either the nucleophile or the electrophile (Scheme 1b). Moreover, since the method utilizes 9-BBN boranes as nucleophiles, the need to use three equivalents of the coupling partner to generate the borane nucleophile is eliminated.

To develop an iron-based catalyst capable of mediating alkyl-alkyl cross-coupling reactions, we started from our previously established system for the crosscoupling of alkyl halides and arylboronic esters utilizing $\beta$-diketiminate iron complexes. ${ }^{5}$ This monoanionic ligand helps prevent iron-aggregates from forming that lead to catalyst death. ${ }^{5}$ Additionally, the steric and electronic environment of the $\beta$-diketiminate ligand is easily modified, which is attractive for developing an alkyl-alkyl Suzuki-Miyaura cross-coupling reaction. Preliminary investigations began with a crosscoupling reaction between (3-bromobutyl)benzene (1) and octyl-B(pin) using lithum methylethylamide as the base. When this coupling reaction was performed in benzene using iron complex 2 as the catalyst, no cross-coupled product $\mathbf{3}$ was detected. Replacing the boronic ester with the more reactive 9-BBN alkylborane 4 and changing the base to lithium dimethylamide resulted in high conversion of $\mathbf{1}$ to crosscoupled product 3 , which was obtained in $75 \%$ yield (Table 1, entry 1). The major byproduct observed in these reactions was alkane $\mathbf{5}$ that results from protodehalogenation of the electrophile. ${ }^{29}$ As we have seen previously, 5,30,31 using presynthesized $\mathbf{2}$ was essential for obtaining high yields of cross-coupled product. Lower yields were obtained when attempting to form the complex in situ by mixing $\mathrm{FeCl}_{2}$ with the b-dekitiminate ligand (entry 2). Similarly, the supporting $\beta$-diketiminate ligand framework was essential for effective cross-coupling because $\mathrm{FeCl}_{2}$ led to very low yields of cross-coupled products (entry 3 ). ${ }^{32}$
Encouraged by these initial results, we next screened iron complexes containing a variety of $\beta$ diketiminate ligands as precatalysts (Table 1). Increasing the steric bulk of the $\beta$-diketiminate using iron complex 6 led to diminished yields of cross-coupled product and a preponderance of organic byproducts (entry 4). ${ }^{32}$ The next logical ligand to test was one containing less bulky substituents installed in the aryl ketimine arms. However, we had limited success in synthesizing such ligands, which precluded their use as catalysts. ${ }^{33}$ Surprisingly, altering the identity of the substituents in the ligand backbone had a profound influence on product yields. Ligands containing the electron-withdrawing $\mathrm{CF}_{3}$ groups in the backbone were essential because replacing them with $\mathrm{CH}_{3}$ groups led to a decreased yield of cross-coupled product 3 from $75 \%$ using 2 (entry 1) to $47 \%$ yield using 7 (entry 5). Similarly, using complex 8 containing two sterically more encumbered tert-butyl substituents in

Table 1. Effect of various $\beta$-diketiminate iron precatalysts on the Suzuki-Miyaura cross-coupling reaction between 3bromobutylbenzene (1) and octyl-9-BBN (4). ${ }^{a}$

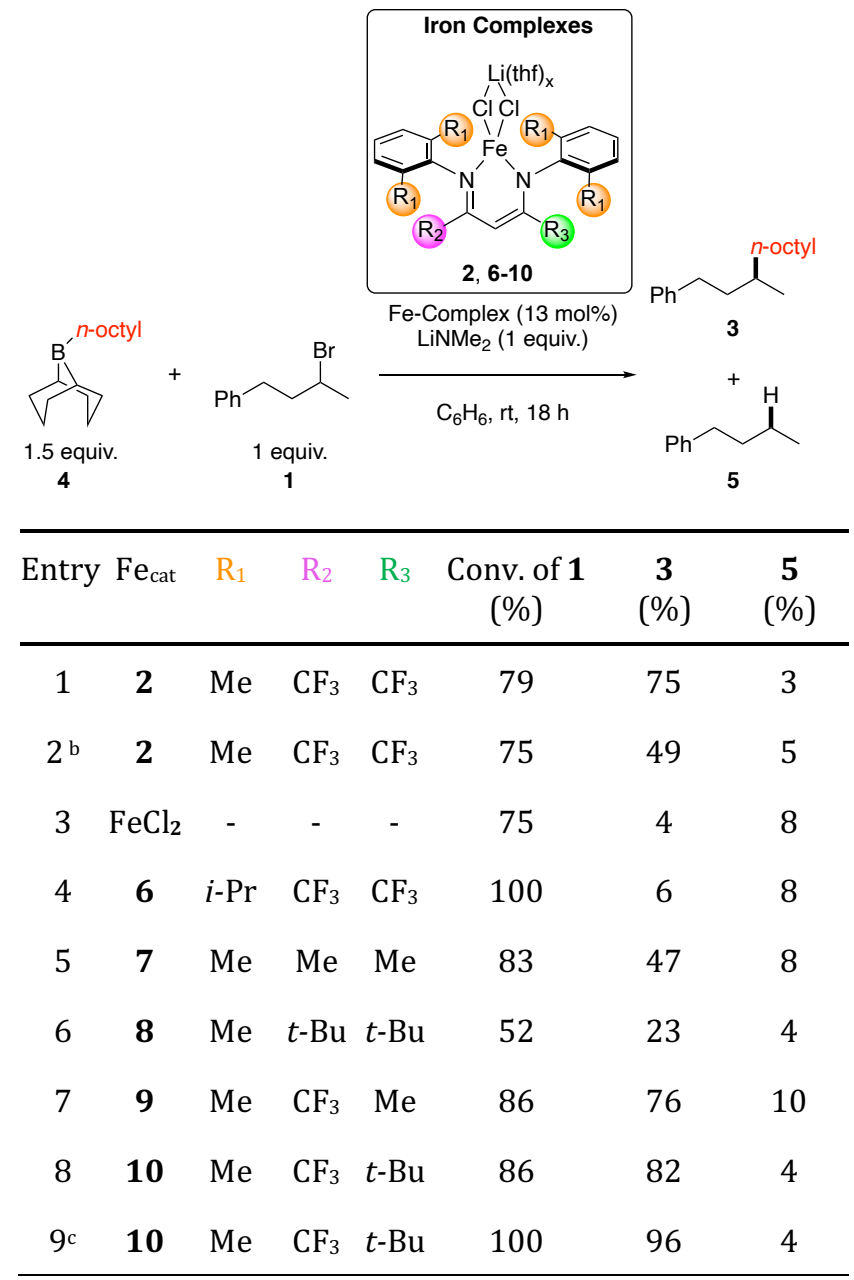

[a] Conversion and product yields are from GC analysis of reaction mixtures relative to a tetradecane internal standard. [b] 2 generated in-situ from $\mathrm{FeCl}_{2}$ and free ligand [c] 2.0 equiv. borane and 1.2 equiv. $\mathrm{LiNMe}_{2}$. 
the ligand backbone led to a marked decrease in crosscoupling yields (entry 6). ${ }^{32}$ In contrast, an iron complex containing dissymmetric $\beta$-diketiminate ligand frameworks with one $\mathrm{CF}_{3}$ group and one alkyl group installed in the ligand backbone proved to be particularly useful to effect high yields in cross-coupling reactions. For example, iron-complex 9 containing a $\mathrm{CF}_{3}$ and $\mathrm{CH}_{3}$ group in the backbone gave nearly identical yields as complex 1 containing two $\mathrm{CF}_{3}$ groups in the $\beta$-diketiminate backbone (entry 7). Replacing the methyl group with a tert-butyl group in the dissymmetric ligand backbone resulted in the iron-complex $\mathbf{1 0}$ that gave the highest yield (82\%) of any iron-complex evaluated (entry 8). Finally, 3 could be isolated in $96 \%$ yield with minimal production of alkane $\mathbf{5}$ and alkene byproducts by using iron complex $\mathbf{1 0}$ as the catalyst and two equivalents of borane and 1.2 equivalents of base (entry 9).

Using the optimized conditions for cross-coupling, the substrate scope of the reaction was evaluated next (Table 2). The reaction was general for a variety of unactivated primary and secondary acyclic alkyl halides, providing high yields of cross-coupled products (e.g., $\mathbf{3}, \mathbf{1 2}, \mathbf{1 3}, \mathbf{1 8}-20,22)$. The reaction was not limited to alkyl bromides as (3-chlorobutyl)benzene was a competent coupling partner producing 3 in 73\% yield. Cyclic secondary alkyl halides were also tolerated, although lower yields where often observed (e.g., 14-17). An exception to this trend was bromocyclohexane, which gave cross-coupled product 17 in $87 \%$ yield. Higher yields of cyclic products $\mathbf{1 5}$ and $\mathbf{1 6}$ could be obtained using less sterically encumbered $C_{1}$-symmetric ironcomplex 11, but this catalyst did not improve yields for $\mathbf{1 4}$ and 15, which are derived from the small, cyclic alkyl halides cyclobutyl bromide and cylcopropyl bromide. While it is clear that the yields are roughly inversely correlated with ring strain in the cyclic alkyl halide, it is not clear at this time why this factor is important.

While there were some limitations with respect to functional group compatibility (e.g. esters, nitriles, see Figure S2) and tertiary alkyl halides did not produce any cross-coupled-product (e.g., 23), we were pleased to see the reaction conditions were tolerant to a variety of other functional groups. Productive cross-coupling was observed for alkyl halide substrates containing acetals (18), silyl-protected alcohols (19), thiophenes (20), Cbz-protected piperidines (21) and $N$ Boc-protected indoles (22). It was particularly exciting to see heteroaromatic-containing alkyl halides produce product efficiently since few of these substrates have been reported in analogous reactions catalyzed by nickel-based complexes. . $^{15} 17$

The utility of the alkyl halide substrate scope prompted us to explore the generality of the alkylborane coupling partners (Table 2). The alkyl halide substrate scope was carried out with two different

Table 2: Substrate scope of the Suzuki-Miyaura cross-coupling reaction between alkyl halide and alkyl-9-BBN catalyzed by $\beta$-diketiminate iron complex 10.

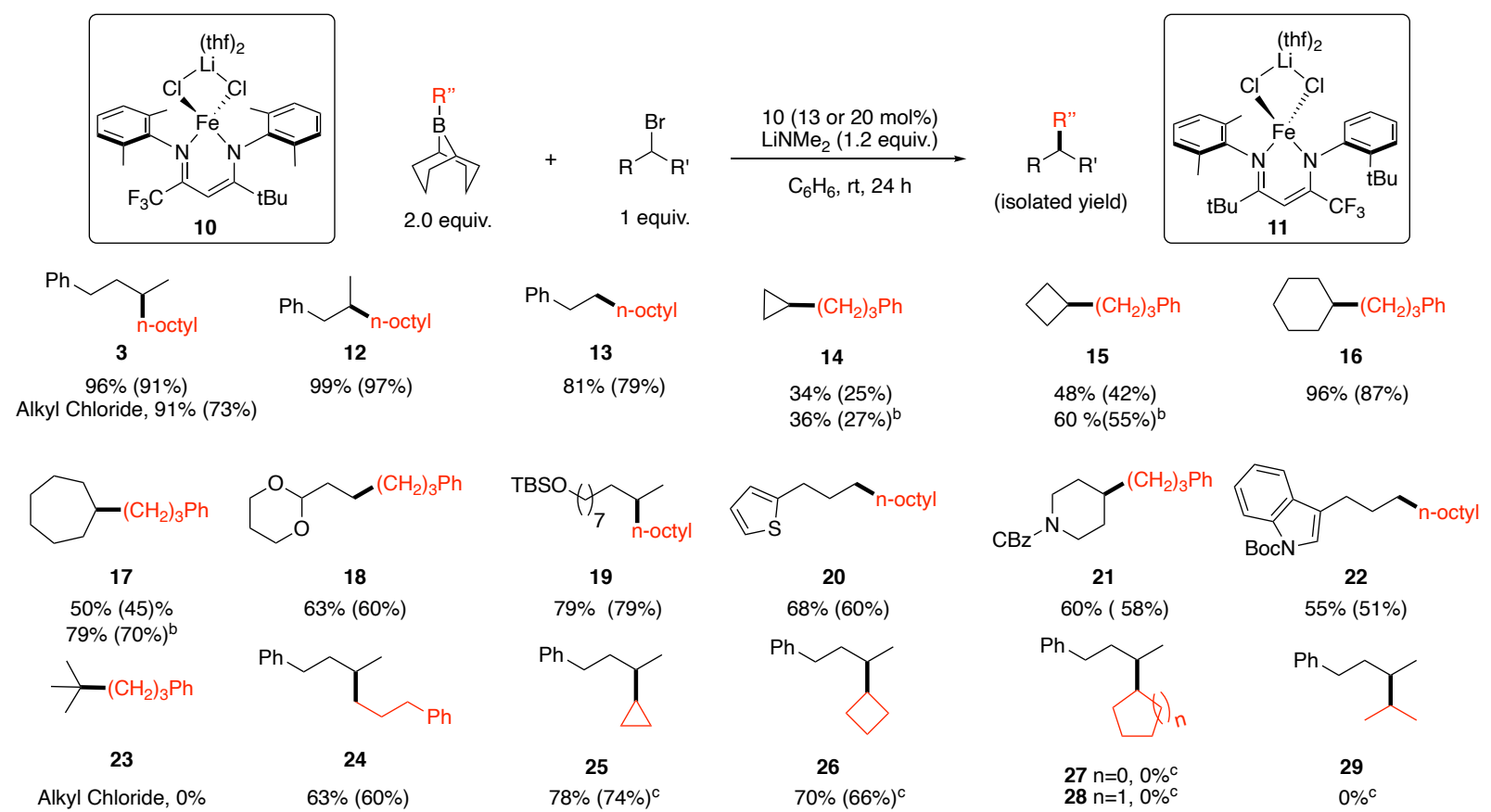

[a] Yields shown are from ${ }^{1} \mathrm{H}-\mathrm{NMR}$ analysis of the reaction mixture relative to 1,3,5-trimethoxybenzene as an internal standard. Yields shown in parentheses indicate product yield after isolation. ${ }^{[b]}$ Complex 11 used. ${ }^{[c]} 20$ mol\% 10 used. 
primary alkylboranes, and high yields of cross-coupled products were generally observed. We anticipated that secondary alkylboranes would be more challenging substrates due to their historical lack of reactivity. ${ }^{14}$ Despite this expectation, when cyclopropyl-9-BBN was used in a cross-coupling reaction using $20 \mathrm{~mol} \%$ of iron complex 11, cross-coupled product 25 was obtained in 74\% yield. Moreover, cyclobutyl9-BBN also led to cross-coupled product 26 in 66\% yield. Unfortunately, bulkier cyclic boranes and an acyclic secondary borane led to no products 27-29. Nevertheless, these results are noteworthy because there are very few examples of $\mathrm{sp}^{3}-\mathrm{sp}^{3}$ cross-coupling reactions using secondary alkyl nucleophiles with most reported examples being Negishi reactions rather than Suzuki-Miyaura reactions. ${ }^{34,35}$

Given the generality that the reaction demonstrated for a variety of alkyl halide and borane substrates, we decided to see if the cross-coupling reaction would be compatible for installing methyl groups in small molecules. Such a capability would be particularly important in the area of medicinal chemistry due to the "magic methyl" effect known to elicit favorable medicinal properties. ${ }^{36}$ Cross-coupling reactions would be a convenient way to install methyl groups into biologically active small molecules at $\mathrm{sp}^{3}$-hybridized sites, but there are only a handful of examples in the literature. ${ }^{37}$ When we carried out methylation reactions using the iron-based complex $\mathbf{1 0}$ and our established protocol for alkyl-alkyl cross-coupling, cross-coupled product 30 could be obtained in $63 \%$ yield when methyl iodide was used as the methylating source. Likewise, when Me-9-BBN was used as the methylating reagent, 31 could be obtained in 54\% yield (Scheme $2 \mathrm{a}$ ). To the best of our knowledge, these coupling-partners have not previously been reported in analogous iron or nickel-catalyzed alkyl-alkyl Suzuki-Miyaura reactions.

The two methylation reactions provide complementary means for installing methyl groups in complex small molecules. To showcase this fact, we decided to functionalize steroids derived from lithocholic acid (Scheme $2 b$ ). ${ }^{38}$ Using a sequence of standard transformations, lithocholic acid derivative $\mathbf{3 2}$ could be converted into alkyl halide $\mathbf{3 3}$ or alkylborane $\mathbf{3 4}$, which could serve as the electrophile and nucleophile in cross-coupling reactions, respectively (Scheme 3). Methylation of 33 using Me-9-BBN led to 35 in 75\% yield. Similarly, methylation of $\mathbf{3 4}$ using methyl iodide led to $80 \%$ of $\mathbf{3 6}$. The two products $\mathbf{3 5}$ and $\mathbf{3 6}$ are isomeric and constitute the formal addition of a methyl group in two different positions to lithocholic acid derivative $\mathbf{3 2}$. Access to these isomers is only made possible because the cross-coupling reaction is compatible with nucleophilic and electrophilic methylating sources.
Scheme 2: a) Complementary methylation reactions using electrophilic and nucleophilic methylating reactants. b) diversification of lithocholic acid using complementary methylating reagents.

a.

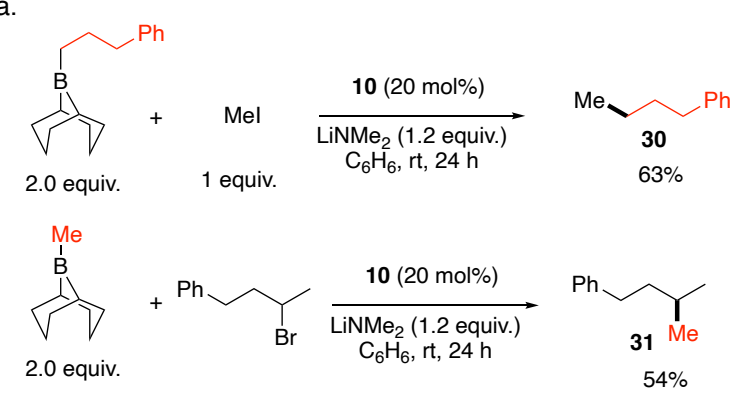

b.

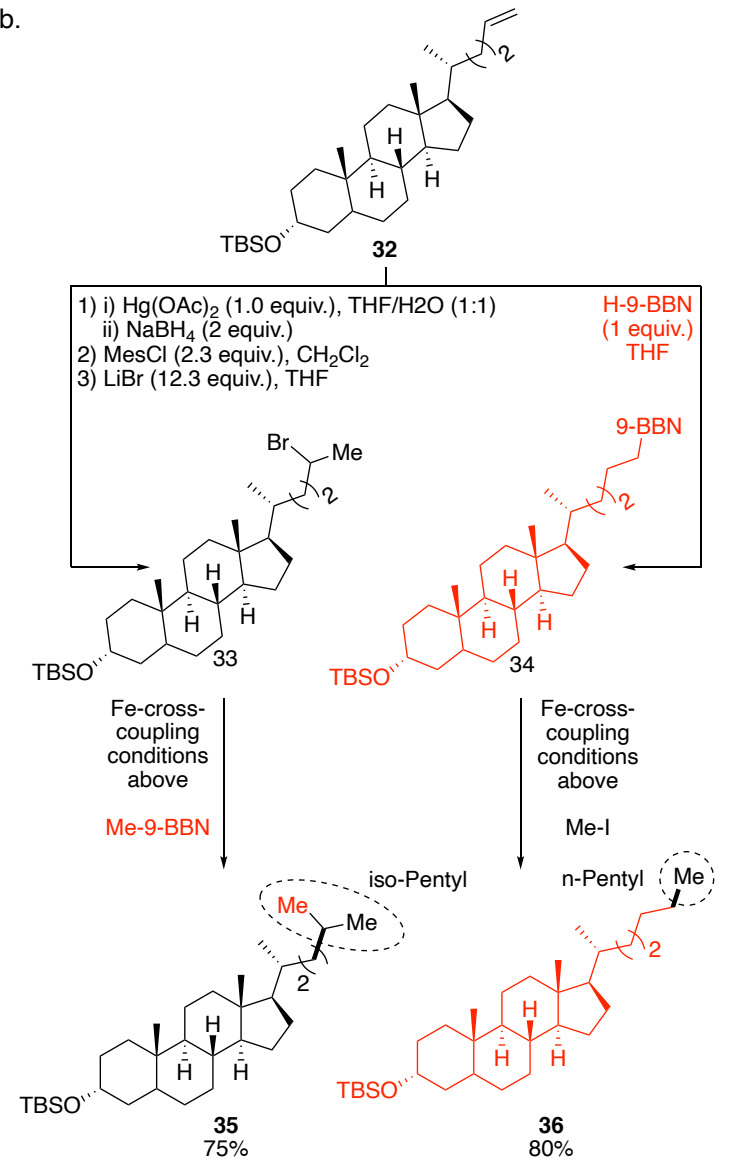

While mechanistic investigations are beyond the scope of this study, our current working mechanistic hypothesis is a bimetallic mechanism similar to what we have proposed previously for alkyl-aryl SuzukiMiyaura cross-coupling reactions (Scheme 3).5,31 In this bimetallic mechanism, iron(II) halide I undergoes salt metathesis with the lithium amide to form iron(II) amide II. This intermediate is poised for transmetalation with the alkylborane to form iron(II) alkyl complex III. It also can carry out halogen abstraction to form a carbon-centered radical and iron(III) amidochloride complex VI. The carbon-centered radical can then recombine with iron(II) alkyl III to form an iron(III) bis-alkyl species IV. Species IV is then poised 
for reductive elimination to furnish cross-coupled product and a putative low valent iron(I) species $\mathbf{V}$. To complete the catalytic cycle, $\mathbf{V}$ comproportionates with iron(III) amido-chloride VI to regenerate I and II. While this mechanism is the one we currently favor, we cannot rule out other reasonable possibilities, such as those that involve only one metal center (See Figures S4-5). Future research will be dedicated to better understanding the mechanism of this transformation and how it relates to similar aryl-alkyl cross-coupling reactions we have developed. 5

Scheme 3: Working mechanistic hypothesis.

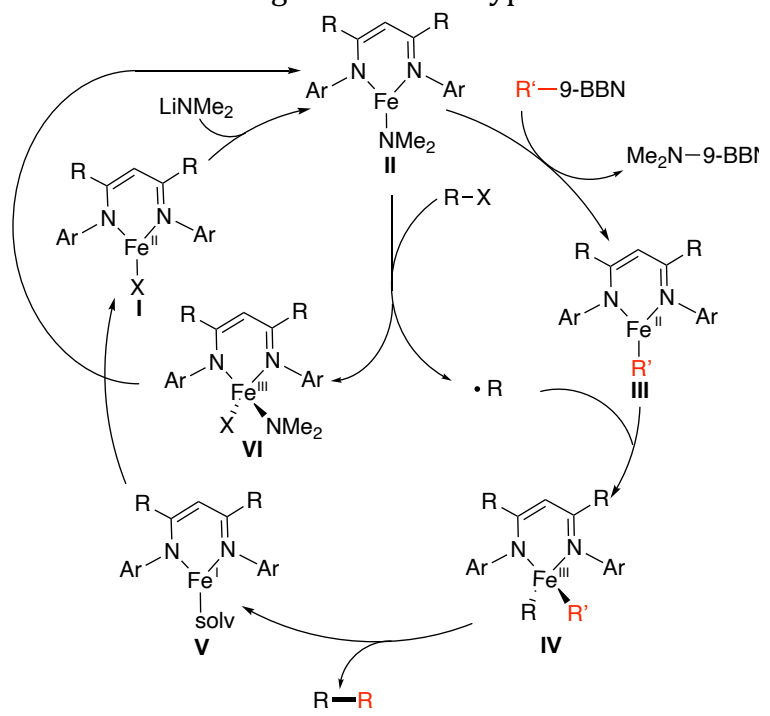

In summary, we have demonstrated an efficient and general alkyl-alkyl Suzuki-Miyaura cross-coupling reaction catalyzed by an iron-based complex. High yields were obtained between primary alkylboranes and a wide array of unactivated alkyl halides, including primary and secondary alkyl bromides and even an alkyl chloride. The reaction was also compatible with different alkylboranes. In particular, we present the first example using cyclobutyl-9-BBN as a coupling partner, which holds promise for future development to access previously unreactive secondary nucleophile coupling-partners. The method is also useful for installing methyl groups, either using electrophilic or nucleophilic methylating reagents, which provide complementary methods to diversify biologically active small molecules. Key to the success of the method was modifying the backbone of the $\beta$-diketiminate ancillary ligand, which proved to be an effective way to finely tune the electronic and steric environment of the ligand to achieve optimal performance. In the future, improved mechanistic understanding of these processes is intended, which is expected to lead to new reaction development including using boronic esters as coupling partners and stereoconvergent alkylalkyl cross-coupling reactions.

\section{ACKNOWLEDGMENT}

This work was funded by the ACS PRF 59542-ND1.

\section{REFERENCES}

(1) Jana, R.; Pathak, T. P.; Sigman, M. S. Advances in Transition Metal (Pd,Ni,Fe)-Catalyzed Cross-Coupling Reactions Using Alkyl-Organometallics as Reaction Partners. Chem. Rev. 2011, 111 (3), 1417-1492. https://doi.org/10.1021/cr100327p.

(2) Brown, D. G.; Boström, J. Analysis of Past and Present Synthetic Methodologies on Medicinal Chemistry: Where Have All the New Reactions Gone? J. Med. Chem. 2016, 59 (10), 4443-4458.

https://doi.org/10.1021/acs.jmedchem.5b01409.
Geist, E.; Kirschning, A.; Schmidt, T. $\mathrm{Sp}^{3}-\mathrm{Sp}^{3}$ Coupling Reactions in the Synthesis of Natural Products and Biologically Active Molecules. Nat. Prod. Rep. 2014, 31 (4), 441-448. https://doi.org/10.1039/c3np70108e.

Choi, J.; Fu, G. C. Transition Metal-Catalyzed Alkyl-Alkyl Bond Formation: Another Dimension in Cross-Coupling Chemistry. Science, 2017, 356, 559-564. https://doi.org/10.1126/science.aaf7230.

Crockett, M. P.; Wong, A. S.; Li, B.; Byers, J. A. Rational Design of an Iron-Based Catalyst for Suzuki-Miyaura CrossCouplings Involving Heteroaromatic Boronic Esters and Tertiary Alkyl Electrophiles. Angew. Chemie Int. Ed. 2020, 59 (13), 5392-5397. https://doi.org/10.1002/anie.201914315.

Netherton, M. R.; Dai, C.; Neuschütz, K.; Fu, G. C. RoomTemperature Alkyl-Alkyl Suzuki Cross-Coupling of Alkyl Bromides That Possess $\beta$ Hydrogens. J. Am. Chem. Soc. 2001, 123 (41), 10099-10100. https://doi.org/10.1021/ja011306o.

Lu, Z.; Fu, G. C. Alkyl-Alkyl Suzuki Cross-Coupling of Unactivated Secondary Alkyl Chlorides. Angew. Chemie Int. Ed. 2010, 49 (37), 6676-6678. https://doi.org/10.1002/anie.201003272.

Liang, Y.; Fu, G. C. Nickel-Catalyzed Alkyl-Alkyl CrossCouplings of Fluorinated Secondary Electrophiles: A General Approach to the Synthesis of Compounds Having a Perfluoroalkyl Substituent. Angew. Chemie Int. Ed. 2015, 54 (31), 9047-9051. https://doi.org/10.1002/anie.201503297.

Lou, S., Fu, G. C. Palladium-Catalyzed Alkyl-Alkyl Suzuki Cross-Couplings of Primary Alkyl Bromides At Room Temperature. Org. Synth. 2010, 87, 299. https://doi.org/10.15227/orgsyn.087.0299.

Saito, B.; Fu, G. C. Alkyl-Alkyl Suzuki Cross-Couplings of Unactivated Secondary Alkyl Halides at Room Temperature. J. Am. Chem. Soc. 2007, 129, 9602-9603. https://doi.org/10.1021/ja0740081.

Jiang, X.; Gandelman, M. Enantioselective Suzuki CrossCouplings of Unactivated 1-Fluoro-1-Haloalkanes: Synthesis of Chiral $\beta-, \gamma-, \delta$-, and $\varepsilon$-Fluoroalkanes. J. Am. Chem. Soc. 2015, 137 (7), 2542-2547. https://doi.org/10.1021/jacs.5b00473.

Lu, Z.; Wilsily, A.; Fu, G. C. Stereoconvergent Amine-Directed Alkyl-Alkyl Suzuki Reactions of Unactivated Secondary Alkyl Chlorides. J. Am. Chem. Soc. 2011, 133 (21), 8154-8157. https://doi.org/10.1021/ja203560q.

Wilsily, A.; Tramutola, F.; Owston, N. A.; Fu, G. C. New Directing Groups for Metal-Catalyzed Asymmetric CarbonCarbon Bond-Forming Processes: Stereoconvergent AlkylAlkyl Suzuki Cross-Couplings of Unactivated Electrophiles. J. 
Am. Chem. Soc. 2012, 134 (13), 5794-5797. https://doi.org/10.1021/ja301612y.

(14)

Zultanski, S. L.; Fu, G. C. Catalytic Asymmetric $\gamma$-Alkylation of Carbonyl Compounds via Stereoconvergent Suzuki CrossCouplings. J. Am. Chem. Soc. 2011, 133 (39), 15362-15364. https://doi.org/10.1021/ja2079515.

Saito, B.; Fu, G. C. Enantioselective Alkyl-Alkyl Suzuki CrossCouplings of Unactivated Homobenzylic Halides. J. Am. Chem. $\begin{array}{lllll}\text { Soc. 2008, } & 130 \quad \text { (21), 6694-6695. }\end{array}$ https://doi.org/10.1021/ja8013677.

Owston, N. A.; Fu, G. C. Asymmetric Alkyl-Alkyl CrossCouplings of Unactivated Secondary Alkyl Electrophiles: Stereoconvergent Suzuki Reactions of Racemic Acylated Halohydrins. J. Am. Chem. Soc. 2010, 132 (34), 11908-11909. https://doi.org/10.1021/ja105924f.

Di Franco, T.; Boutin, N.; Hu, X. Suzuki-Miyaura CrossCoupling Reactions of Unactivated Alkyl Halides Catalyzed by a Nickel Pincer Complex. Synth. 2013, 45 (21), 2949-2958. https://doi.org/10.1055/s-0033-1338544.

(18) Egorova, K. S.; Ananikov, V. P. Toxicity of Metal Compounds: Knowledge and Myths. Organometallics 2017, 36 (21), 40714090. https://doi.org/10.1021/acs.organomet.7b00605.

(19) Furstner, A.; Leitner, A.; Mndez, M.; Krause, H. Iron-Catalyzed Cross-Coupling Reactions Iron-Catalyzed Cross-Coupling Reactions. Tetrahedron 2002, 124, 13856-13863. https://doi.org/10.1021/ja027190t.

Wang, X.; Wang, S.; Xue, W.; Gong, H. Nickel-Catalyzed Reductive Coupling of Aryl Bromides with Tertiary Alkyl Halides. J. Am. Chem. Soc. 2015, 137 (36), 11562-11565. https://doi.org/10.1021/jacs.5b06255.

Wang, X.; Ma, G.; Peng, Y.; Pitsch, C. E.; Moll, B. J.; Ly, T. D.; Wang, X.; Gong, H. Ni-Catalyzed Reductive Coupling of Electron-Rich Aryl Iodides with Tertiary Alkyl Halides. J. Am. Chem. Soc. 2018, 140 (43), 14490-14497. https://doi.org/10.1021/jacs.8b09473.

(22) Cherney, A. H.; Hedley, S. J.; Mennen, S. M.; Tedrow, J. S. Xantphos as a Branch-Selective Ligand for the Acyclic SecAlkyl Negishi Cross-Coupling of Heteroaryl Halides. Organometallics 2019, 38 (1), 97-102. https://doi.org/10.1021/acs.organomet.8b00590.

(23) Zultanski, S. L.; Fu, G. C. Nickel-Catalyzed Carbon-Carbon Bond-Forming Reactions of Unactivated Tertiary Alkyl Halides: Suzuki Arylations. J. Am. Chem. Soc. 2013, 135 (2), 624-627. https://doi.org/10.1021/ja311669p.

Dongol, K. G.; Koh, H.; Sau, M.; Chai, C. L. L. Iron-Catalysed Sp³ $\mathrm{Sp}^{3}$ Cross-Coupling Reactions of Unactivated Alkyl Halides with Alkyl Grignard Reagents. Adv. Synth. Catal. 2007, 349 (7), 1015-1018. https://doi.org/10.1002/adsc.200600383.

Guisán-Ceinos, M.; Tato, F.; Buñuel, E.; Calle, P.; Cárdenas, D. J. Fe-Catalysed Kumada-Type Alkyl-Alkyl Cross-Coupling. Evidence for the Intermediacy of Fe(i) Complexes. Chem. Sci. 2013, 4 (3), 1098-1104. https://doi.org/10.1039/c2sc21754f.

(26) Iwasaki, T.; Shimizu, R.; Imanishi, R.; Kuniyasu, H.; Kambe, N. Cross-Coupling Reaction of Alkyl Halides with Alkyl Grignard Reagents Catalyzed by Cp-Iron Complexes in the Presence of 1,3-Butadiene. Chem. Lett. 2018, 47 (6), 763-766. https://doi.org/10.1246/cl.180201.
(28) Hatakeyama, T.; Hashimoto, T.; Kathriarachchi, K. K. A. D. S.; Zenmyo, T.; Seike, H.; Nakamura, M. Iron-Catalyzed AlkylAlkyl Suzuki-Miyaura Coupling. Angew. Chemie - Int. Ed. 2012 51 (35), 8834-8837. https://doi.org/10.1002/anie.201202797.

Hexadecane was also formed as a byproduct from homodimerization of $n$-octyl-9-BBN in 1-8\% yields.

(30) Crockett, M. P.; Tyrol, C. C.; Wong, A. S.; Li, B.; Byers, J. A. IronCatalyzed Suzuki-Miyaura Cross-Coupling Reactions between Alkyl Halides and Unactivated Arylboronic Esters. Org. Lett. 2018, $20 \quad$ (17), 5233-5237. https://doi.org/10.1021/acs.orglett.8b02184.

(31) Tyrol, C. C.; Yone, N. S.; Gallin, C. F.; Byers, J. A. Iron-Catalysed Enantioconvergent Suzuki-Miyaura Cross-Coupling to Afford Enantioenriched 1,1-Diarylalkanes. Chem. Commun. 2020, 56 (93), 14661-14664. https://doi.org/10.1039/d0cc05003b.

(32) We were not able to quantify the yields of the organic byproducts originating from $\mathbf{1}$, but we presume some byproducts are alkene isomers, resulting from $ß$-hydride elimination.

(33) We were able to synthesize a ligand with two $\mathrm{CF}_{3}$ groups installed in the backbone and containing 2-t-butyl aryl ketemine arms in small quantities. This complex resulted in $78 \%$ of 3 , similar to yields obtained with the easier to synthesize 2 .

(34) Cordier, C. J.; Lundgren, R. J.; Fu, G. C. Enantioconvergent Cross-Couplings of Racemic Alkylmetal Reagents with Unactivated Secondary Alkyl Electrophiles: Catalytic Asymmetric Negishi $\alpha$-Alkylations of N-Boc-Pyrrolidine. J. Am. Chem. Soc. 2013, 135 (30), 10946-10949. https://doi.org/10.1021/ja4054114.

Mu, X.; Shibata, Y.; Makida, Y.; Fu, G. C. Control of Vicinal Stereocenters through Nickel-Catalyzed Alkyl-Alkyl CrossCoupling. Angew. Chemie - Int. Ed. 2017, 56 (21), 5821-5824. https://doi.org/10.1002/anie.201702402.

(36) Schönherr, H.; Cernak, T. Profound Methyl Effects in Drug Discovery and a Call for New C-H Methylation Reactions. Angew. Chemie Int. Ed. 2013, 52 (47), 12256-12267. https://doi.org/10.1002/anie.201303207.

(37) Gee, A.; Windhorst, A. Pd-Mediated Rapid Cross-Couplings Using [ $11 \mathrm{C}$ ] Methyl Iodide : Groundbreaking Labeling. 2015, 58 (3), 73-85. . https://doi.org/10.1002/jlcr.3253.

(38) Fernández-cabezón, L.; Galán, B.; García, J. L. New Insights on Steroid Biotechnology. 2018, 9, 1-15. https://doi.org/10.3389/fmicb.2018.00958. 
Insert Table of Contents artwork here

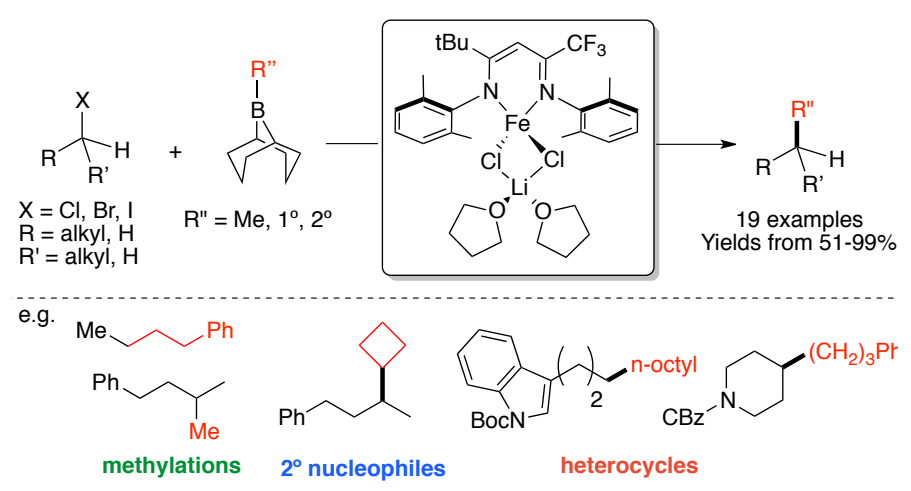

